\title{
Fighting the battle of the bulge: exercise training in early-onset type 2 diabetes
}

\author{
M. E. Osler • J. R. Zierath
}

Published online: 8 May 2007

(C) Springer-Verlag 2007

Keywords Beta cell function - Early-onset - Exercise intervention - Glucose metabolism - Insulin resistance . Type 2 diabetes · Visceral obesity · Young

\begin{abstract}
Abbreviations
$V \mathrm{O}_{2 \max }$ maximum volume of oxygen utilisation
\end{abstract}

As we enter the twenty-first century, alarming clinical and epidemiological statistics reflect the shift to a sedentary lifestyle, as technology increasingly replaces any remaining daily requirement to be active. The role of visceral obesity in the development of insulin resistance and type 2 diabetes, and of other diabetes-related disorders, is underscored by the rising trend of physical inactivity and overnutrition. Obesity often lays the foundation for the development of type 2 diabetes and other linked metabolic disorders, including cardiovascular disease and cancer, and is becoming excessively common in the young. For example, the prevalence of obesity in adolescents aged 12-19 years in the USA increased from 5.0 to $17.4 \%$ between the late 1970s and 2004 [1]. Unchecked, this striking rise in childhood obesity will increase the prevalence of fatal and non-fatal diseases, and the steady rise in

\footnotetext{
M. E. Osler · J. R. Zierath $(\bowtie)$

Department of Molecular Medicine and Surgery,

Section of Integrative Physiology, Karolinska Institutet,

von Eulers väg 4, 4th floor,

S-171 77 Stockholm, Sweden

e-mail: Juleen.Zierath@ki.se
}

life expectancy over the past two centuries may come to a halt or even be reversed.

In this issue of Diabetologia, Burns et al. [2] shed new light on the impact of obesity in young people with earlyonset type 2 diabetes, and raise intriguing questions regarding the efficacy of exercise training for improvement of insulin sensitivity in this population. Their study determined the effect of adiposity in young, obese, insulin-resistant individuals with and without type 2 diabetes, and investigated whether exercise training improves insulin-mediated glucose metabolism. Obese individuals with diabetes exhibited increases in WHR, fasting triacylglycerol levels and systolic blood pressure compared with the obese non-diabetic individuals, and additionally showed marked reductions in glucose disposal and beta cell function, as reflected by an increase in basal hepatic glucose output. The authors considered two different explanations for these metabolic impairments: (1) that early-onset type 2 diabetes is identical to later-onset type 2 diabetes in terms of pathogenesis and clinical progression; and (2) that it is a more extreme phenotype that is associated with an even greater risk of lifelong cardiovascular and metabolic complications than late-onset diabetes. While this point is not resolved in the present work, their data support previous observations that young people with type 2 diabetes generally have a loss of beta cell insulin secretion, as do older type 2 diabetic patients, at the time of clinical presentation. Moreover, those who develop type 2 diabetes before the age of 40 years are more obese at an earlier age than their older counterparts, have a more deleterious cardiovascular risk profile, and have worse initial and ongoing glycaemic control, despite comparable clinical care [3]. Collectively, these findings support the concept that early-onset type 2 diabetes reflects 
an extreme phenotype. Follow-up studies in early-onset type 2 diabetic patients will reveal whether this population will suffer a disproportionate cardiovascular and metabolic risk burden in the future.

Burns et al. [2] went on to investigate whether modest exercise could improve metabolic defects in early-onset type 2 diabetes, testing the hypothesis that a 12 week exercise training programme would improve insulin sensitivity in both cohorts. Exercise is a leading strategy in the battle against the bulge, and enhances peripheral insulin sensitivity [4]. Acute exercise can increase glucose uptake and metabolism in skeletal muscle via an insulin-independent mechanism that bypasses insulin signalling defects associated with type 2 diabetes [5]. Furthermore, the expression of metabolic and mitochondrial genes involved in glucose and lipid metabolism is increased in a coordinated manner in response to habitual exercise training, which has a positive effect upon whole-body glucose homeostasis [6]. Burns et al. [2] found that non-diabetic obese individuals responded to the exercise training programme with a $20 \%$ increase in their maximum volume of oxygen utilisation $\left(\mathrm{VO}_{2 \max }\right)$. Waist circumference was decreased in the early-onset type 2 diabetic patients after exercise training, but all other clinical parameters were unchanged. Furthermore, there was a greater increase in glucose disposal and a greater reduction in fasting NEFA in the non-diabetic obese group than in the diabetic group, despite identical compliance with the training programme. These findings contrast with previous reports in older individuals with type 2 diabetes or obesity, in whom exercise triggers some level of metabolic improvement in both groups [7]. Burns et al. [2] thus provide new evidence that earlyonset obese type 2 diabetic patients are less responsive to the clinical benefits of exercise training.

The idea that there are exercise responders and nonresponders is not new, but is perhaps underappreciated. In a microarray analysis of skeletal muscle biopsies from the HERITAGE Family Study, Teran-Garcia et al. [8] demonstrated differences in gene expression profiles between groups that do or do not exhibit improvements in insulin sensitivity following a 20 week training protocol, and propose that a differentially expressed set of genes may predict the exercise training response. In a study similar to that of Burns et al. [2], involving an older population [9], exercise intervention reversed clinical and metabolic consequences of type 2 diabetes. Fritz et al. [9] compared the expression of genes involved in glucose and lipid metabolism in the skeletal muscle of middle-aged type 2 diabetic patients after a 4 month walking programme. They found that exercise responders, who demonstrated a marked improvement in clinical variables, glucose metabolism, triacylglycerol and $V \mathrm{O}_{2 \max }$, differed from the non-responders in the expression of metabolic genes. These findings suggest that intrinsic genetic differences may dictate the exercise response and metabolic adaptation in skeletal muscle. Moreover, compelling work from Kelley et al. [10] suggests that exercise in obese/diabetic individuals boosts mitochondrial function [6]. Such findings spark further questions regarding the identification of biomarkers that may be useful in defining responder and non-responder phenotypes.

Burns et al. [2] are among the first to address the effectiveness of an intervention study in early-onset type 2 diabetic subjects aged between 15 and 30 years. The lack of clear benefits from exercise raises additional questions as to the mechanism underlying the failure to respond, and further emphasises the multifaceted phenotype of obesity coupled to type 2 diabetes. Although the authors did not perform a gene expression analysis, the possibility that differential transcript profiles play a role in segregating responders from nonresponders is attractive and requires attention. Alternatively, these findings may imply that early-onset obesity and/or type 2 diabetes could shift the metabolic equilibrium beyond the reach of an activity-based improvement (Fig. 1), and cast doubt on the assumption that an exercise training protocol can improve insulin sensitivity in every metabolic situation. It is also possible that the conversion of an early-onset exercise non-responder to a responder may simply involve a more aggressive exercise intervention protocol.

Regardless, this study should not distract attention from the countless individual examples showing that exercise restores dysfunctional metabolic responses and that the benefit of exercise training in the management and

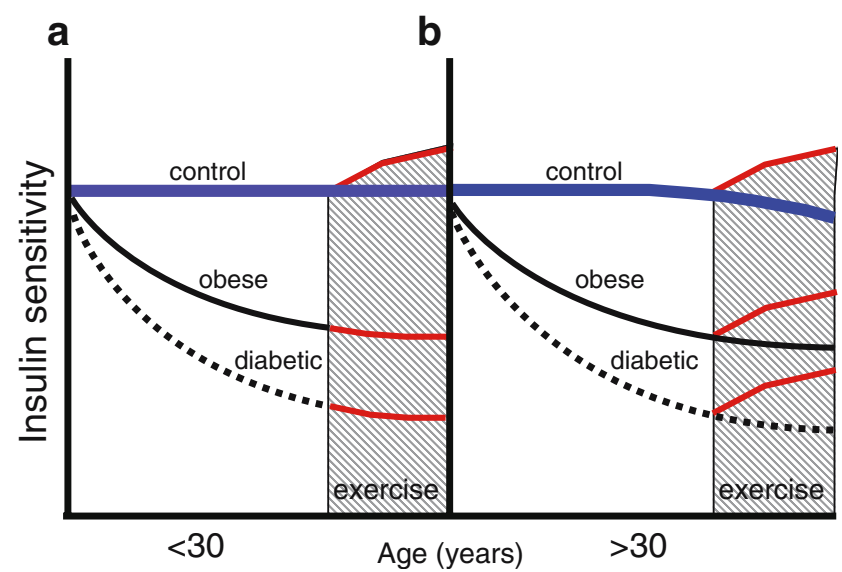

Fig. 1 Age-graded response to aerobic exercise in obese and type 2 diabetic patients. Independent of the age of onset, obesity and diabetes result in a progressive decrease in insulin sensitivity over time (black lines). Exercise intervention (red lines) improves insulin sensitivity and promotes weight loss in states of later-onset ( $>30$ years) obesity and type 2 diabetes (b). This response mimics the observed effects in the non-obese, non-diabetic population (control). Early-onset obesity and type 2 diabetes (a) may render a state of 'exercise unresponsiveness' upon initiation of a physical training programme 
prevention of type 2 diabetes is supported by a substantial body of evidence [4]. Further intervention studies will be required to determine which variables, such as duration, time, distance, aerobic nature and involvement of resistance training, elicit the most useful metabolic responses, and the influence of genetic factors upon the heterogeneity of exercise responsiveness.

In summary, Burns et al. [2] have shown that there are still unresolved issues regarding an appropriate exercise prescription to combat insulin resistance in obesity and type 2 diabetes. Although teasing out the ideal exercise protocol may not be a rapid process, physical activity remains the better option for treatment of metabolic disorders in most cases. A recent review points out that 'although the magnitude of the effect of exercise on body fatness might be modest in most individuals, exercise can nevertheless be an important public health measure to reduce the prevalence of overweight [individuals]' [11]. Moreover, the concept of NEAT (nonexercise activity thermogenesis), as introduced by Levine et al. [12], appears to be an important component in weight management as the 'principal mediator of resistance to fat gain with overfeeding'. In short, daily activity does not have to be performed entirely in the form of a rigid exercise regimen; leisure-time physical activity such as gardening or household chores can also play a useful role in warding off obesity. On present evidence, a two-pronged attack seems justified. First, through meticulous design of future intervention studies in the young obese population, we must identify the best strategy to counteract the potential long-term health burden of type 2 diabetes. Second, emphasis should be placed on a more forceful and profound educational push for lifestyle changes and early intervention. In the words of the Chinese proverb, 'The journey of a thousand miles begins with a single step.'

\section{References}

1. National Health and Nutrition Examination Survey (2005) NHANES data on the prevalence of overweight among children and adolescents: United States, 2003-2004 Centers for Disease Control and Prevention, National Center for Health Statistics, Health E-Stats, Hyattsville, USA

2. Burns N, Finucane FM, Hatunic M et al (2007) Early-onset type 2 diabetes in obese white subjects is characterised by a marked defect in beta cell insulin secretion, severe insulin resistance and a lack of response to aerobic exercise training. Diabetologia DOI 10.1007/s00125-007-0655-7

3. Hatunic M, Burns N, Finucane F, Mannion C, Nolan JJ (2005) Contrasting clinical and cardiovascular risk status between early and later onset type 2 diabetes. Diab Vasc Dis Res 2:73-75

4. Hawley JA (2004) Exercise as a therapeutic intervention for the prevention and treatment of insulin resistance. Diabetes Metab Res Rev 20:383-393

5. Zierath JR, Krook A, Wallberg-Henriksson H (2000) Insulin action and insulin resistance in human skeletal muscle. Diabetologia 43:821-835

6. Menshikova EV, Ritov VB, Fairfull L, Ferrell RE, Kelley DE, Goodpaster BH (2006) Effects of exercise on mitochondrial content and function in aging human skeletal muscle. J Gerontol A Biol Sci Med Sci 61:534-540

7. Eriksson J, Lindstrom J, Valle T et al (1999) Prevention of type II diabetes in subjects with impaired glucose tolerance: the Diabetes Prevention Study (DPS) in Finland. Study design and 1-year interim report on the feasibility of the lifestyle intervention programme. Diabetologia 42:793-801

8. Teran-Garcia M, Rankinen T, Koza RA, Rao DC, Bouchard C (2005) Endurance training-induced changes in insulin sensitivity and gene expression. Am J Physiol Endocrinol Metab 288:E1168-E1178

9. Fritz T, Kramer DK, Karlsson HK (2006) Low-intensity exercise increases skeletal muscle protein expression of PPAR $\delta$ and UCP3 in type 2 diabetic patients. Diabetes Metab Res Rev 22:492-498

10. Kelley DE, He J, Menshikova EV, Ritov VB (2002) Dysfunction of mitochondria in human skeletal muscle in type 2 diabetes. Diabetes 51:2944-2950

11. Elder SJ, Roberts SB (2007) The effects of exercise on food intake and body fatness: a summary of published studies. Nutr Rev 65:1-19

12. Levine JA, Eberhardt NL, Jensen MD (1999) Role of nonexercise activity thermogenesis in resistance to fat gain in humans. Science 283:212-214 